\title{
ON THE IRREDUCIBILITY OF CERTAIN POLYNOMIALS
}

\section{ALFRED BRAUER AND GERTRUDE EHRLICH}

Introduction. G. Pólya ${ }^{1}$ has proved the following theorem:

If for $n$ integral values of $x$, the integral polynomial $P(x)$ of degree $n$ has values which are different from zero and, without regard to sign, less than

$$
G_{1}=\frac{(n-[n / 2]) !}{2^{n-[n / 2]}},
$$

then $P(x)$ is irreducible in the field of rational numbers. (Here, as in the following, a polynomial with rational integral coefficients is called an "integral polynomial.")

This result was improved for positively definite polynomials by Hildegard Ille ${ }^{2}$ and for arbitrary polynomials by T. Tatuzawa. ${ }^{3}$ The latter obtained the larger bound

$$
G_{2}=\left(2^{-n}(n-1) !\right)^{1 / 2}
$$

instead of $G_{1}$. Moreover he proved the following theorem:

If for $l$ integral values of $x$ where $n>l>n / 2$, the integral polynomial $P(x)$ of degree $n$ takes values which are different from zero and, without regard to sign, less than

$$
H_{1}=(l-1) !^{1 / l} / 2,
$$

then $P(x)$ is irreducible in the field of rational numbers.

In the following, the results of Tatuzawa will be improved further by a slight modification of his method. Instead of $G_{2}$ we obtain the larger bound

We have

$$
G=\frac{(n-1) !}{2^{n-1}[(n-2) / 2] !} .
$$

$$
\frac{G}{G_{2}} \sim\left(\frac{2 n}{\pi}\right)^{1 / 4} \text { for even } n \text { and } \quad \frac{G}{G_{2}} \sim\left(\frac{n^{3}}{2 \pi}\right)^{1 / 4} \text { for odd } n .
$$

Presented to the Society, April 27, 1946; received by the editors November 12, 1945.

1 Verschiedene Bemerkungen zur Zahlentheorie, Jber. Deutschen Math. Verein. vol. 28 (1919) pp. 31-40.

${ }^{2}$ Einige Bemerkungen zu einem von G. Polya herrilhrenden Irreduzibilitätskriterium, Jber. Deutschen Math. Verein. vol. 35 (1926) pp. 204-208.

3 Über die Irreduzibilität gewisser ganzzahliger Polynome, Proc. Imp. Acad. Tokyo vol. 15 (1939) pp. 253-254. 
It follows from this theorem in particular that the integral polynomials

$$
P(x)=A\left(x-x_{1}\right)\left(x-x_{2}\right) \cdots\left(x-x_{n}\right)+t
$$

are irreducible if $x_{\mu} \neq x_{\nu}$ and $1 \leqq|t|<G$. This result contains for $n>4$, $A=1$, and $t= \pm 1$ a theorem of I. Schur, ${ }^{4}$ and for arbitrary $A$ and $t= \pm 1$ a theorem of H. L. Dorwart and O. Ore. ${ }^{5}$ For $n \leqq 4$ there are exceptions. ${ }^{6}$ The application of Pólya's bound gives this result only for $n>6$, that of Tatuzawa for $n>5$, while our bound gives the exact degrees for which these theorems hold.

Moreover, we improve the second theorem of Tatuzawa. Instead of $H_{1}$ we obtain the larger bound

$$
H=\left[\frac{l+1}{2}\right] \text { for } l \geqq 7, \quad H=\frac{3}{2} \text { for } l=6 \text { and } 5 .
$$

It is of interest that this bound cannot be improved further. If the absolute value of $P\left(x_{\lambda}\right)$ for $\lambda=1,2, \cdots, 1$ is not less than $H$, but only less than or equal to $H$, then our result does not remain correct. It will be shown that for every $n>2$ such polynomials exist which are reducible in the field of rational numbers.

It follows from our theorem that the integral polynomials of degree $n$

$$
P(x)=\left(x-x_{1}\right)\left(x-x_{2}\right) \cdots\left(x-x_{l}\right) h(x)+t
$$

are irreducible if $l>4, n>l>n / 2, x_{\lambda} \neq x_{\mu}$, and $1 \leqq|t|<H$. This gives for $t= \pm 1$ a theorem of Dorwart and Ore. ${ }^{7}$

Finally a criterion of a new type is obtained. $P(x)$ is irreducible if the absolute value of $P\left(x_{v}\right)$ is different from zero and less than a certain bound $S_{1}>G$ for $n$ different integers $x_{v}$, but less than another smaller constant $S_{2}$ for $l$ of these $x_{\nu}$. More exactly, the following theorem is proved.

Let $P(x)$ be an integral polynomial of degree $n$; let $k, l$, and $h$ be integers satisfying the following conditions: $k \geqq[(n+1) / 2], n>l$ $>n / 2, l>12$ or $l=11$ or 9 ,

$$
l>h, \quad n \geqq k+h-1 .
$$

4 Aufgabe 226, Archiv der Mathematik und Physik (3) vol. 13 (1908) p. 367. Lösung by W. Flügel, ibid. vol. 15 (1909) pp. 271-272. Cf. G. P6lya and G. Szegö, Aufgaben und Lehrsätze aus der Analysis, vol. 2, Berlin, 1925, pp. 136, 346-347.

${ }_{5}$ Criteria for the irreducibility of polynomials, Ann. of Math. vol. 34 (1933) pp. 81-94, 195.

6 See, for instance, loc. cit. footnote 5, pp. 86, 195.

7 Loc. cit. footnote 5. 
If for $n$ different integers $x_{1}, x_{2}, \cdots, x_{n}$

$$
0<\left|P\left(x_{\nu}\right)\right|<2^{-k} \prod_{k=1}^{k}\left[\frac{\kappa n-1}{k}\right] \quad(\nu=1,2, \cdots, n),
$$

and for $l$ of these $x_{\nu}$, say $x^{(1)}, x^{(2)}, \cdots, x^{(l)}$

$$
\left|P\left(x^{(\lambda)}\right)\right|<2^{-h} \prod_{\mu=1}^{h}\left[\frac{\mu l-1}{h}\right] \quad(\lambda=1,2, \cdots, l),
$$

then $P(x)$ is irreducible in the field of rational numbers.

1. Bounds for the absolute value of polynomials at given points. We first prove the following theorem of Tatuzawa.

TheOREM 1. Let $f(x)=a_{0} x^{k}+a_{1} x^{k-1}+\cdots+a_{k}$ be a polynomial of degree $k$, let $x_{1}<x_{2}<\cdots<x_{k+1}$ be arbitrary numbers, and $d_{\kappa}$ the length of the smallest interval which contains $\kappa+1$ of these numbers $(\kappa=1,2$, $\cdots, k)$. Then

$$
\max _{\kappa=1,2, \cdots, k+1}\left|f\left(x_{\kappa}\right)\right| \geqq 2^{-k}\left|a_{0}\right| d_{1} d_{2} \cdots d_{k} .
$$

Proof. For $k=1$ we have

$$
\left|f\left(x_{1}\right)-f\left(x_{2}\right)\right|=\left|a_{0}\left(x_{1}-x_{2}\right)\right|=\left|a_{0}\right| d_{1}
$$

hence

$$
\begin{aligned}
\max \left\{\left|f\left(x_{1}\right)\right|,\left|f\left(x_{2}\right)\right|\right\} & \geqq 2^{-1}\left\{\left|f\left(x_{1}\right)\right|+\left|f\left(x_{2}\right)\right|\right\} \\
& \geqq 2^{-1}\left|f\left(x_{1}\right)-f\left(x_{2}\right)\right|=2^{-1}\left|a_{0}\right| d_{1} .
\end{aligned}
$$

This proves the theorem for $k=1$. Let us now assume that it is already proved for polynomials of degree less than $k$.

We divide $f(x)$ by the polynomials $\left(x-x_{1}\right)\left(x-x_{2}\right) \cdots\left(x-x_{k}\right)$ and $\left(x-x_{2}\right)\left(x-x_{3}\right) \cdots\left(x-x_{k+1}\right)$, respectively. Then

$$
\begin{aligned}
f(x) & =a_{0}\left(x-x_{1}\right)\left(x-x_{2}\right) \cdots\left(x-x_{k}\right)+g(x) \\
& =a_{0}\left(x-x_{2}\right)\left(x-x_{3}\right) \cdots\left(x-x_{k+1}\right)+h(x)
\end{aligned}
$$

where $g(x)$ and $h(x)$ are polynomials of degree less than $k$ with the highest coefficients

and

$$
b_{0}=a_{1}+a_{0}\left(x_{1}+x_{2}+\cdots+x_{k}\right)
$$

$$
c_{0}=a_{1}+a_{0}\left(x_{2}+x_{3}+\cdots+x_{k+1}\right),
$$

respectively, hence $b_{0}-c_{0}=a_{0}\left(x_{1}-x_{k+1}\right)$, 


$$
\begin{aligned}
\max \left(\left|b_{0}\right|,\left|c_{0}\right|\right) & \geqq 2^{-1}\left(\left|b_{0}\right|+\left|c_{0}\right|\right) \\
& \geqq 2^{-1}\left|b_{0}-c_{0}\right|=2^{-1}\left|a_{0}\right| d_{k} .
\end{aligned}
$$

On the other hand it follows from (1) that

$$
\begin{aligned}
\max _{\kappa=1,2, \cdots, k+1}\left|f\left(x_{\kappa}\right)\right| \geqq \max _{\kappa=1,2, \cdots, k}\left|g\left(x_{\kappa}\right)\right|, \\
\max _{\kappa=1,2, \cdots, k+1}\left|f\left(x_{\kappa}\right)\right| \geqq \max _{\kappa=2,3, \cdots, k+1}\left|h\left(x_{\kappa}\right)\right| .
\end{aligned}
$$

Since $g(x)$ and $h(x)$ are of lower degree than $k$, our theorem may be applied to them. The lengths of the smallest intervals which contain $\kappa$ of the points $x_{1}, x_{2}, \cdots, x_{k}$ or $\kappa$ of the points $x_{2}, x_{3}, \cdots, x_{k+1}$ are both not smaller than $d_{k-1}$. Hence

$$
\begin{aligned}
& \max _{\kappa=1,2, \cdots, k}\left|g\left(x_{\kappa}\right)\right| \geqq 2^{-k+1}\left|b_{0}\right| d_{1} d_{2} \cdots d_{k-1}, \\
& \max _{\kappa=2,3, \cdots, k+1}\left|h\left(x_{\kappa}\right)\right| \geqq 2^{-k+1}\left|c_{0}\right| d_{1} d_{2} \cdots d_{k-1},
\end{aligned}
$$

and by (3) and (2)

$$
\begin{aligned}
\max _{\kappa=1,2, \cdots, k+1}\left|f\left(x_{\kappa}\right)\right| & \geqq 2^{-k+1} d_{1} d_{2} \cdots d_{k-1}\left\{\max \left(\left|b_{0}\right|,\left|c_{0}\right|\right)\right\} \\
& =2^{-k}\left|a_{0}\right| d_{1} d_{2} \cdots d_{k} .
\end{aligned}
$$

THEOREM 2. Let $f(x)=a_{0} x^{k}+a_{1} x^{k-1}+\cdots+a_{k}$ be a polynomial of degree $k$ and $x_{1}<x_{2}<\cdots<x_{n}$ a set of more than $k$ integers. Then

$$
\max _{\nu=1,2, \cdots, n}\left|f\left(x_{\nu}\right)\right| \geqq 2^{-k}\left|a_{0}\right| \prod_{k=1}^{k}\left[\frac{\kappa n-1}{k}\right] .
$$

PrOOF. We consider those $k+1$ of the integers $x_{\nu}$ whose subscripts are 1 and the $k$ numbers

$$
1+\left[\frac{\rho n-1}{k}\right] \quad(\rho=1,2, \cdots, k),
$$

and denote these $x_{\nu}$ in increasing order by $x_{0}^{\prime}, x_{1}^{\prime}, \cdots, x_{k}^{\prime}$. The difference of two consecutive elements $x_{\nu}$ is at least 1 , hence for $k \geqq \beta>\alpha>0$

$$
x_{\beta}^{\prime}-x_{\alpha}^{\prime} \geqq\left[\frac{\beta n-1}{k}\right]-\left[\frac{\alpha n-1}{k}\right] \text {. }
$$

Since for every $r$ and $s$

we have

$$
\left[\frac{r+s}{k}\right] \geqq\left[\frac{r}{k}\right]+\left[\frac{s}{k}\right],
$$




$$
\left[\frac{\beta n-1}{k}\right] \geqq\left[\frac{(\beta-\alpha) n-1}{k}\right]+\left[\frac{\alpha n}{k}\right],
$$

hence by (4)

$$
\begin{aligned}
x_{\beta}^{\prime}-x_{\alpha}^{\prime} & \geqq\left[\frac{(\beta-\alpha) n-1}{k}\right]+\left[\frac{\alpha n}{k}\right]-\left[\frac{\alpha n-1}{k}\right] \\
& \geqq\left[\frac{(\beta-\alpha) n-1}{k}\right] .
\end{aligned}
$$

It is obvious that (5) holds also for $\alpha=0$. If the length of the smallest interval which contains $\kappa+1$ of the numbers $x_{0}^{\prime}, x_{1}^{\prime}, \cdots, x_{k}^{\prime}$ is denoted by $d_{k}^{\prime}$, then by (5)

hence, by Theorem 1 ,

$$
d_{\kappa}^{\prime} \geqq\left[\frac{\kappa n-1}{k}\right] \quad(\kappa=1,2, \cdots, k),
$$

$$
\max _{\nu=1,2, \cdots, n}\left|f\left(x_{\nu}\right)\right| \geqq \max _{k=0,1, \cdots, k}\left|f\left(x_{k}^{\prime}\right)\right| \geqq 2^{-k}\left|a_{0}\right| \prod_{k=1}^{k}\left[\frac{\kappa n-1}{k}\right] .
$$

2. Criteria for irreducibility. Theorem 2 will be used now to obtain criteria for irreducibility of polynomials.

THEOREM 3. Let $P(x)$ be a polynomial of degree $n$ with integral rational coefficients. If for $n$ integral values $x_{1}, x_{2}, \cdots, x_{n}$ the absolute value of $P\left(x_{\nu}\right)$ for $\nu=1,2, \cdots, n$ is less than

$$
G=\frac{(n-1) !}{2^{n-1}\{[(n-2) / 2] !\}},
$$

but different from 0 , then $P(x)$ is irreducible in the field of rational numbers.

Proof. If $P(x)$ is reducible, then it contains a factor $f(x)$ of degree $k$ with integral coefficients where $n>k \geqq[(n+1) / 2]$. It follows now from Theorem 2 that

$$
M=\max _{\nu=1,2, \cdots, n}\left|f\left(x_{\nu}\right)\right| \geqq 2^{-k} \prod_{\kappa=1}^{k}\left[\frac{\kappa n-1}{k}\right] .
$$

We set for fixed $n$

$$
\phi(k)=\phi(k, n)=2^{-k} \prod_{k=1}^{k}\left[\frac{\kappa n-1}{k}\right] .
$$

Let us first assume that $k=[(n+1) / 2]$. For even values of $n$ we have 


$$
\left[\frac{\kappa n-1}{k}\right]=\left[\frac{\kappa n-1}{n / 2}\right]=\left[\frac{2 \kappa n-2}{n}\right]=2 \kappa-1 \quad(\kappa=1,2, \cdots, k),
$$

hence by (7)

$$
\phi\{[(n+1) / 2]\} \geqq 2^{-k} \prod_{\kappa=1}^{k}(2 \kappa-1)=2^{-n / 2} \cdot 1 \cdot 3 \cdot 5 \cdots(n-1)
$$

$$
=\frac{(n-1) !}{2^{n / 2+(n-2) / 2}[(n-2) / 2] !}=\frac{(n-1) !}{2^{n-1}\{[(n-2) / 2] !\}}=G .
$$

If $n$ is odd, then

hence

$$
\begin{aligned}
{\left[\frac{\kappa n-1}{k}\right] } & =\left[\frac{\kappa n-1}{(n+1) / 2}\right]=\left[\frac{2 \kappa n-2}{n+1}\right] \\
& =\left[\frac{(2 \kappa-1)(n+1)+n-2 \kappa-1}{n+1}\right],
\end{aligned}
$$

$$
\left[\frac{\kappa n-1}{k}\right]= \begin{cases}2 \kappa-1 & \text { for } \quad \kappa=1,2, \cdots,(n-1) / 2 \\ 2 \kappa-2 & \text { for } \quad \kappa=(n+1) / 2\end{cases}
$$

and by (7)

(9)

$$
\begin{gathered}
\phi\{[(n+1) / 2]\} \geqq 2^{-(n+1) / 2}(n-1) \prod_{\kappa=1}^{(n-1) / 2}(2 \kappa-1) \\
=\frac{(n-1) !}{2^{(n+1) / 2+(n-3) / 2}\{[(n-3) / 2] !\}}=\frac{(n-1) !}{2^{n-1}\{[(n-2) / 2] !\}}=G .
\end{gathered}
$$

It follows from (8) and (9) that

$$
\phi\left(\left[\frac{n+1}{2}\right]\right) \geqq G .
$$

Now we maintain that

$$
\phi(k+1) \geqq \phi(k) \text { for }\left[\frac{n+1}{2}\right] \leqq k \leqq n-2 .
$$

For this purpose we want to prove that

$$
\left[\frac{(\kappa+1) n-1}{k+1}\right] \geqq\left[\frac{\kappa n-1}{k}\right] \quad(\kappa=2,3, \cdots, k) .
$$

We divide $\kappa n-1$ by $k$ : 


$$
\kappa n-1=q k+r \quad(0 \leqq r<k),
$$

hence $\kappa n>q k$ and $n>q$ since $\kappa \leqq k$. It follows that

$$
\begin{aligned}
{\left[\frac{(\kappa+1) n-1}{k+1}\right] } & =\left[\frac{q k+r+n}{k+1}\right]=\left[\frac{q(k+1)+r+n-q}{k+1}\right] \\
& \geqq q=\left[\frac{k n-1}{k}\right] .
\end{aligned}
$$

This proves (12). Moreover we have for $\kappa=1$

$$
\left[\frac{2 n-1}{k+1}\right] \geqq\left[\frac{2 n-1}{n-1}\right]=2=2\left[\frac{n-1}{k}\right]
$$

because it is sufficient for the proof of (11) to assume that $k+1 \leqq n-1$ and $k \geqq[(n+1) / 2]$. Multiplying (13) and (12) for $\kappa=2,3, \cdots, k$ we obtain

$$
\prod_{\kappa=1}^{k}\left[\frac{(\kappa+1) n-1}{k+1}\right] \geqq 2 \prod_{\kappa=1}^{k}\left[\frac{\kappa n-1}{k}\right],
$$

hence, since $[(n-1) /(k+1)]=1$,

$$
\begin{aligned}
2^{-k-1} \prod_{k=1}^{k+1}\left[\frac{\kappa n-1}{k+1}\right] & =2^{-k-1}\left[\frac{n-1}{k+1}\right] \prod_{\kappa=1}^{k}\left[\frac{(\kappa+1) n-1}{k+1}\right] \\
& \geqq 2^{-k} \prod_{k=1}^{k}\left[\frac{\kappa n-1}{k}\right] .
\end{aligned}
$$

This proves (11). It follows now from (6), (7), (11), and (10) that

$$
M \geqq G \text {. }
$$

Since $P(x) / f(x)$ is a polynomial with integral coefficients and $P\left(x_{\nu}\right)$ $\neq 0$, we obtain

$$
\max _{\nu=1,2, \cdots, n}\left|P\left(x_{\nu}\right)\right| \geqq \max _{\nu=1,2, \cdots, n}\left|f\left(x_{\nu}\right)\right|=M \geqq G .
$$

This contradicts our assumption, and the theorem is proved.

The bound of Tatuzawa is

$$
G_{2}=\left(2^{-n}(n-1) !\right)^{1 / 2}
$$

and our bound by (8) and (9)

$$
G=\frac{(n-1) !}{2^{n-1}\{[(n-2) / 2] !\}}=\left\{\begin{array}{lc}
2^{-n / 2} \cdot 1 \cdot 3 \cdot 5 \cdots(n-1) & \text { for even } n, \\
2^{-(n+1) / 2}(n-1) \cdot 1 \cdot 3 \cdot 5 \cdots(n-2) & \text { for odd } n .
\end{array}\right.
$$


Now we have by the formula of Wallis

$$
\frac{1 \cdot 3 \cdot 5 \cdots(2 m-1)}{2 \cdot 4 \cdot 6 \cdots(2 m-2)} \sim\left(\frac{4 m}{\pi}\right)^{1 / 2} .
$$

For even $n$ we obtain

$$
\frac{G}{G_{2}}=\left(\frac{1 \cdot 3 \cdot 5 \cdots(n-1)}{2 \cdot 4 \cdot 6 \cdots(n-2)}\right)^{1 / 2} \sim\left(\frac{2 n}{\pi}\right)^{1 / 4},
$$

and for odd $n$

$\frac{G}{G_{2}}=\frac{1}{2^{1 / 2}}\left(\frac{(n-1) \cdot 1 \cdot 3 \cdot 5 \cdots(n-2)}{2 \cdot 4 \cdot 6 \cdots(n-3)}\right)^{1 / 2} \sim\left(\frac{n}{2}\right)^{1 / 2}\left(\frac{2 n}{\pi}\right)^{1 / 4}=\left(\frac{n^{3}}{2 \pi}\right)^{1 / 4}$.

Moreover, we have $G>G_{2}$ for $n>3$.

CoROLlary. The integral polynomials

$$
A\left(x-x_{1}\right)\left(x-x_{2}\right) \cdots\left(x-x_{n}\right)+t
$$

are irreducible in the field of rational numbers if $x_{\mu} \neq x_{\nu}$ and $1 \leqq|t|<G$.

We have $G>1$ for $n>4$. Therefore the polynomials (14) are irreducible for $t= \pm 1$ and $n>4$. This is, as already mentioned in the introduction, for $A=1$ a theorem of Schur, and for arbitrary $A$ a theorem of Dorwart and Ore.

We can formulate Theorem 3 also in the following form:

TheOREM 3a. Let $P(x)$ be an integral polynomial of degree $n$. If for $n$ different integers $x_{1}, x_{2}, \cdots, x_{n}$

$$
0<\left|P\left(x_{\nu}\right)\right|<2^{-k} \prod_{k=1}^{k}\left[\frac{\kappa n-1}{k}\right]=\phi(k) \quad(\nu=1,2, \cdots, n)
$$

where $k \geqq[(n+1) / 2]$, then $P(x)$ cannot contain a factor $f^{*}(x)$ of degree $k^{*}$ with $k \leqq k^{*}<n$.

Proof. If $P(x)$ contains such a factor, then by (6), (7), and (11)

$$
\max _{\nu=1,2, \cdots, n}\left|P\left(x_{\nu}\right)\right| \geqq \max _{\nu=1,2, \cdots, n}\left|f^{*}\left(x_{\nu}\right)\right| \geqq \phi\left(k^{*}\right) \geqq \phi(k) .
$$

This contradicts our assumption.

Theorem 4. Let $P(x)$ be a polynomial of degree $n$ with integral coefficients, $l$ an integer with $l \geqq 5$, and $n>l>n / 2$. If for $l$ different integers $x_{1}, x_{2}, \cdots, x_{l}$

$$
0<\left|P\left(x_{\lambda}\right)\right|<H \quad(\lambda=1,2, \cdots, l)
$$


where $H=[(l+1) / 2]$ for $l \geqq 7, H=3 / 2$ for $l=6$ and 5 , then $P(x)$ is irreducible in the field of rational numbers.

Proof. If $P(x)$ is reducible, then it must contain a factor $g(x)$ of degree $k$ less than or equal to $n / 2$ with integral coefficients. Here $k>1$. For a linear polynomial takes each value only once, hence the $l$ integers $g\left(x_{1}\right), g\left(x_{2}\right), \cdots, g\left(x_{l}\right)$ must be different. However, only the $2[(l-1) / 2]$ values $\pm 1, \pm 2, \cdots, \pm[(l-1) / 2]$ are possible because

$$
0<\left|g\left(x_{\lambda}\right)\right| \leqq\left|P\left(x_{\lambda}\right)\right|<H \leqq\left[\frac{l+1}{2}\right] \quad(\lambda=1,2, \cdots, l) .
$$

This gives a contradiction since $l>2[(l-1) / 2]$.

It follows from Theorem 2 that

$$
\max _{\lambda=1,2, \cdots, l}\left|g\left(x_{\lambda}\right)\right| \geqq 2^{-k} \prod_{k=1}^{k}\left[\frac{k l-1}{k}\right] .
$$

We denote the right-hand side of (15), for a given $l$, similarly as in (10), by $\phi(k)$ and maintain that

(16) $\phi(k+1) \geqq \phi(k)$ for $2 \leqq k \leqq(l-3) / 2$ and for $l / 2 \leqq k \leqq l-2$.

It follows from the proof of Theorem 3 that (16) holds for $l / 2 \leqq k$ $\leqq l-2$ if we write $l$ instead of $n$ since $[(l+1) / 2]=l / 2$ for even $l$ and $k \neq l / 2$ for odd $l$.

Now we consider the case $k \leqq(l-3) / 2$. Here we have

$$
\left[\frac{l-1}{k+1}\right] \geqq\left[\frac{l-1}{(l-1) / 2}\right]=2 .
$$

Moreover, it follows from (12) that

$$
\left[\frac{(\kappa+1) l-1}{k+1}\right] \geqq\left[\frac{\kappa l-1}{k}\right] \quad(\kappa=1,2, \cdots, k) .
$$

We proved (12) only for $\kappa \geqq 2$; but the proof remains correct for $\kappa=1$. It follows now from (18) and (17) that

$$
\begin{aligned}
2^{-k-1} \prod_{k=0}^{k}\left[\frac{(\kappa+1) l-1}{k+1}\right] & =2^{-k-1} \prod_{k=1}^{k+1}\left[\frac{k l-1}{k+1}\right] \\
& \geqq 2^{-k} \prod_{k=1}^{k}\left[\frac{\kappa l-1}{k}\right],
\end{aligned}
$$

hence $\phi(k+1) \geqq \phi(k)$ for $k \leqq(l-3) / 2$, and (16) is proved.

We now consider the remaining value $k=[(l-1) / 2]$. We maintain that also here 


$$
\phi(k+1) \geqq \phi(k) \text { for } l>12 \text { and } l=11,9 .
$$

For even $l$ we have $k=(l-2) / 2$. It follows from (7) and (8) that for $l>12$

and

$$
\begin{aligned}
\phi(k+1) & =\phi\left\{\left[\frac{l+1}{2}\right]\right\} \geqq 2^{-l / 2} \cdot 1 \cdot 3 \cdot 5 \cdot 7 \cdot \prod_{k=5}^{k+1}\left[\frac{\kappa l-1}{k+1}\right] \\
& =2^{-l / 2} \cdot 1 \cdot 3 \cdot 5 \cdot 7 \cdot \prod_{k=4}^{k}\left[\frac{(\kappa+1) l-1}{k+1}\right]
\end{aligned}
$$

$$
\begin{aligned}
\phi(k) & =\phi\left\{\left[\frac{l-1}{2}\right]\right\} \\
& =2^{-(l-2) / 2}\left[\frac{l-1}{(l-2) / 2}\right]\left[\frac{2 l-1}{(l-2) / 2}\right]\left[\frac{3 l-1}{(l-2) / 2}\right] \prod_{k=4}^{k}\left[\frac{k l-1}{k}\right] \\
& =2^{-(l-2) / 2}\left[\frac{2 l-2}{l-2}\right]\left[\frac{4 l-2}{l-2}\right]\left[\frac{6 l-2}{l-2}\right] \prod_{k=4}^{k}\left[\frac{\kappa l-1}{k}\right] .
\end{aligned}
$$

Now $[(6 l-2) /(l-2)]=6$ since $6 l-2<7(l-2)$ for $l>12$. Similarly $[(2 l-2) /(l-2)]=2$ and $[(4 l-2) /(l-2)]=4$, hence

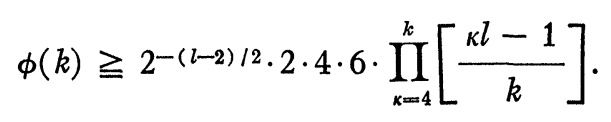

Since $3 \cdot 5 \cdot 7>2 \cdot 2 \cdot 4 \cdot 6$, it follows from (20), (21), and (12) that (19) holds for even $l>12$.

Now, let $l$ be odd. Here we have by (7) and (9) for $l>7$

and

$$
\begin{aligned}
& \phi(k+1)=\phi\left(\frac{l+1}{2}\right)=2^{-(l+1) / 2} \cdot 1 \cdot 3 \cdot 5 \cdot 7 \cdot \prod_{k=5}^{k+1}\left[\frac{k l-1}{k+1}\right] \\
& =2^{-(l+1) / 2} \cdot 1 \cdot 3 \cdot 5 \cdot 7 \cdot \prod_{k=4}^{k}\left[\frac{(\kappa+1) l-1}{k+1}\right]
\end{aligned}
$$

$$
\begin{aligned}
\phi(k) & =\phi\left(\frac{l-1}{2}\right) \\
& =2^{-(l-1) / 2}\left[\frac{2 l-2}{l-1}\right]\left[\frac{4 l-2}{l-1}\right]\left[\frac{6 l-2}{l-1}\right] \prod_{k=4}^{k}\left[\frac{\kappa l-1}{k}\right] \\
& =2^{-(l-1) / 2} \cdot 2 \cdot 4 \cdot 6 \cdot \prod_{k=4}^{k}\left[\frac{\kappa l-1}{k}\right]
\end{aligned}
$$


since $6 l-2<7(l-1)$. It follows from (22), (23), and (12) that (19) holds for odd $l>7$, too.

Now we have by (16) and (19)

$$
\phi(k+1) \geqq \phi(k) \text { for } 2 \leqq k \leqq l-2 \text {, and } l>12, l=11,9 .
$$

By direct calculation we obtain

$$
\begin{aligned}
& \phi(5)>\phi(6)>\phi(4) \quad \text { for } l=12, \\
& \phi(4)>\phi(5)>\phi(3) \text { for } l=10, \\
& \phi(3)>\phi(4)>\phi(2) \text { for } l=8 \text { and } l=7 .
\end{aligned}
$$

By (24) and (25) we have for $l \geqq 7$

$$
\phi(k) \geqq \phi(2)=\left[\frac{2 l-1}{2}\right]\left[\frac{l-1}{2}\right]>\left[\frac{l+1}{2}\right]=H \quad(3 \leqq k<l),
$$

and by direct calculation

$$
\phi(k) \geqq 3 / 2=H \text { for } l=6 \text { and } 5 \quad(2 \leqq k<l),
$$

hence, by (15), (26), and (27),

$$
\max _{\lambda=1,2, \cdots, l}\left|P\left(x_{\lambda}\right)\right| \geqq \max _{\lambda=1,2, \cdots, l}\left|g\left(x_{\lambda}\right)\right| \geqq \phi(k) \geqq H .
$$

This contradicts our assumption, and $P(x)$ must be irreducible.

Theorem 4 cannot be improved further. If we assume instead of

$$
0<\left|P\left(x_{\lambda}\right)\right|<[(l+1) / 2] \quad(\lambda=1,2, \cdots, l)
$$

only

$$
0<\left|P\left(x_{\lambda}\right)\right| \leqq[(l+1) / 2] \quad(\lambda=1,2, \cdots, l),
$$

then $P(x)$ may be reducible.

This is shown by the following examples

$$
\begin{aligned}
& P(x)=x\left\{h(x) \prod_{\lambda=1}^{l / 2}\left(x^{2}-\lambda^{2}\right)+1\right\} \text { for even } l \\
& P(x)=x\left\{h(x)\left(x-\frac{l+1}{2}\right) \prod_{\lambda=1}^{(l-1) / 2}\left(x^{2}-\lambda^{2}\right)+1\right\} \text { for odd } l
\end{aligned}
$$

where $h(x)$ is an arbitrary integral polynomial of degree $n-l-1$. We have here $P(x)=x$ for $x= \pm 1, \pm 2, \cdots, \pm l / 2$ for even $l$, and $x= \pm 1, \pm 2, \cdots, \pm(l-1) / 2,+(l+1) / 2$ for odd $l$. At $l$ integral points these polynomials take values which are different from zero and, without regard to sign, less than or equal to $[(l+1) / 2]$; but they are reducible. 
CoRollary. Let $h(x)$ be an arbitrary integral polynomial of degree $n-1$ and $x_{1}, x_{2}, \cdots, x_{l}$ different integers. The integral polynomial $P(x)$ of degree $n$,

$$
P(x)=\left(x-x_{1}\right)\left(x-x_{2}\right) \cdots\left(x-x_{l}\right) h(x)+t,
$$

is irreducible in the field of rational numbers if $l \geqq 5, n>l>n / 2$ and $1 \leqq|t|<H$ where $H=[(l+1) / 2]$ for $l \geqq 7$ and $H=3 / 2$ for $l=6$ and 5 .

For the proof of Theorem 4 for $l>12$ it is sufficient to prove only (26) instead of (24). We proved here (24) in order to obtain the following theorem.

THEOREM 4a. Let $P(x)$ be an integral polynomial of degree $n$; let $h$ and $l$ be integers satisfying the following conditions:

$$
n>l>n / 2, \quad l>h \geqq 2, \quad \text { and } \quad l>12 \text {, or } l=11,9 \text {. }
$$

If for $l$ different integers $x_{1}, x_{2}, \cdots, x_{l}$

$$
0<\left|P\left(x_{\lambda}\right)\right|<\phi(h)=2^{-h} \prod_{\mu=1}^{h}\left[\frac{\mu l-1}{h}\right] \text {, }
$$

then $P(x)$ cannot contain a factor of degree $h^{*}$ with $h \leqq h^{*} \leqq n / 2$.

Proof. If $P(x)$ contains a factor of degree $h^{*}$, then by (28) and (24)

$$
\max _{\lambda=1,2, \cdots, \imath}\left|P\left(x_{\lambda}\right)\right| \geqq \max _{\lambda=1,2, \cdots, l}\left|g\left(x_{\lambda}\right)\right| \geqq \phi\left(h^{*}\right) \geqq \phi(h) .
$$

This contradicts our assumption.

A similar theorem can be obtained for $l=12,10,8$, and 7 .

By combining Theorems $3 \mathrm{a}$ and $4 \mathrm{a}$ the following theorem is obtained.

THEOREM 5. Let $P(x)$ be an integral polynomial of degree $n$, and $k$, $l$, and $h$ integers satisfying the following conditions:

$$
\begin{gathered}
k \geqq[(n+1) / 2], \quad n>l>n / 2, l>12 \text { or } l=11 \text { or } 9, \\
l>h, \quad n \geqq k+h-1 .
\end{gathered}
$$

If for $n$ different integers $x_{1}, x_{2}, \cdots, x_{n}$

$$
0<\left|P\left(x_{\nu}\right)\right|<2^{-k} \prod_{k=1}^{k}\left[\frac{\kappa n-1}{k}\right] \quad(\nu=1,2, \cdots, n),
$$

and for $l$ of these $x_{\nu}$, say $x^{(1)}, x^{(2)}, \cdots, x^{(l)}$, 


$$
\left|P\left(x^{(\lambda)}\right)\right|<2^{-h} \prod_{\mu=1}^{h}\left[\frac{\mu l-1}{h}\right] \quad(\lambda=1,2, \cdots, l),
$$

then $P(x)$ is irreducible in the field of rational numbers.

Proof. If $P(x)$ is reducible, then

$$
P(x)=f(x) \cdot g(x) .
$$

If the degree of $f(x)$ is $k^{*} \geqq n / 2$, then the degree of $g(x)$ is $n-k^{*}$. It follows from Theorem 3 a that $k^{*} \leqq k-1$, and from Theorem 4 a that $n-k^{*} \leqq h-1$, hence $n \leqq k+h-2$. This gives a contradiction.

University of North Carolina 